\title{
No benefits from exercise training and worse outcomes in heart failure patients with permanent atrial fibrillation and cardiovascular implantable electronic devices
}

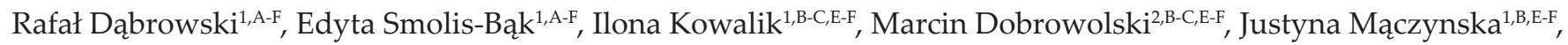
Laura Załucka ${ }^{1, \mathrm{~B}, \mathrm{E}-\mathrm{F}}$, Hanna Rymuza ${ }^{1, \mathrm{~A}-\mathrm{C}, \mathrm{E}-\mathrm{F}}$, Barbara Jędrzejczyk ${ }^{1, \mathrm{~B}-\mathrm{C}, \mathrm{E}-\mathrm{F}}$, Maciej Sterliński ${ }^{1, \mathrm{~B}-\mathrm{C}, \mathrm{E}-\mathrm{F}}$, Mariusz Pytkowski1 ${ }^{1, \mathrm{~B}, \mathrm{E}-\mathrm{F}}$ Hanna Szwed ${ }^{3 \mathrm{~A}-\mathrm{C}, \mathrm{E}-\mathrm{F}}$

A - Research concept and design, B - Collection and/or assembly of data, C - Data analysis and interpretation,

D - Writing the article, E - Critical revision of the article, F - Final approval of article

1. Institute of Cardiology, Warsaw, Poland

2. Kardiologie/Innere Medizin I Department, Helios Klinikum, Schleswig, Germany

3. $2^{\text {nd }}$ Clinic of Coronary Artery Disease, Warsaw, Poland

Address for correspondence:

Rafał Dąbrowski, Institute of Cardiology, Warsaw, Poland

email: rdabrowski@ikard.pl

Edyta Smolis-Bąk, Institute of Cardiology, Warsaw, Poland

email: esmolis@gmail.com

Ilona Kowalik, Institute of Cardiology, Warsaw, Poland

email: ikowalik@ikard.pl

Marcin Dobrowolski, Kardiologie/Innere Medizin I Department, Helios Klinikum, Schleswig, Germany

email: mdobrowolski@pl.pl

Justyna Mączynska, Institute of Cardiology, Warsaw, Poland

email: jmaczynska@ikard.pl

Laura Załucka, Institute of Cardiology, Warsaw, Poland

email: lzalucka@gmail.com,

Hanna Rymuza, Institute of Cardiology, Warsaw, Poland

email: hrymuza@ikard.pl

Barbara Jędrzejczyk, Institute of Cardiology, Warsaw, Poland

email: bjedrzejczyk@ikard.pl

Maciej Sterliński, Institute of Cardiology, Warsaw, Poland

email: msterlinski@poczta.onet.pl

Mariusz Pytkowski, Institute of Cardiology, Warsaw, Poland

email: mpytkowski@ikard.pl

Hanna Szwed, $2^{\text {nd }}$ Clinic of Coronary Artery Disease, Warsaw, Poland

email: hszwed@ikard.pl

Received: 2018-10-02

Revised: 2018-10-19

Accepted: 2018-10-20

Final review: 2018-10-20

DOI: $10.24255 / \mathrm{hbj} / 99092$

\section{Key words:}

ICD, atrial fibrillation, exercise training, CRT-D.

\section{Abstract}

Background: Exercise training (ExT) in patients with heart failure and reduced ejection fraction (HFrEF) improves clinical condition and prognosis. Data are scarce regarding the efficacy and safety of ExT in patients with HFrEF and atrial fibrillation
(AFib) and with a cardiac resynchronization therapy defibrillator (CRT-D) or an implantable cardioverter-defibrillator (ICD).

Purpose: The aim of the study was to evaluate the impact of exercise training in patients with HFrEF (ejection fraction 
$[\mathrm{EF}]<35 \%$ ), in NYHA class III, with AFib, and with a CRT-D or ICD, on the clinical condition and prognosis.

Methods: The study included consecutive patients with a CRT-D or ICD hospitalized due to exacerbation of HFrEF and further randomly assigned to a training program, starting in hospital and continuing in ambulatory conditions for six months. At discharge and after six months, ergospirometry tests (cardiopulmonary exercise testing, CPX) and standard echo examinations were performed. All patients received optimal drug therapy. They were further observed for an average of 54 months and with follow-up visits every 3-6 months. The patients with permanent AFib were analyzed.

Results: 124 patients were enrolled in the study. In this group $39.5 \%$ of patients $(n=49)$ had permanent AFib and $60.5 \%$ $(n=75)$ were in sinus rhythm. Mean age of AFib patients was higher: $65.3 \pm 7.8$ vs. $60.7 \pm 8.2$ years, $p=0.0022$, and more of them had diabetes. Ischemic etiology of HFrEF in AFib patients was most prevalent $(61.2 \%)$. There were no differences in the proportion of subjects in the training program with AFib $(44.9 \%)$ and with SR $(50.7 \%, n s)$. After six months most of the $\mathrm{CPX}$ parameters in the AFib group did not change, but were worse than in the SR group in spite of left ventricular EF improvement. In a 54-month follow-up the overall survival rate was 0.75 and was significantly higher among the 75 patients who did not have AFib (0.80) than among the 49 patients with AFib (0.63, log-rank $p=0.0098)$. Of the 77 patients who had a CRT-D implanted, the survival rate was 0.60 in the AFib group, and 0.83 in the no-AFib group (log-rank $p=0.0259$ ). Of the 47 patients who had an ICD implanted, survival rates were the same regardless of the presence of AFib $(0.66,0.67$, log-rank $\mathrm{p}=0.58$ ). Mortality independent risk factors in multivariate logistic analysis were female gender (adjusted OR 13.2, 95\% CI 1.95-86.1, $\mathrm{p}=0.008$ ) and AFib (adjusted OR 5.13, 95\% CI 1.45$18.1 \mathrm{p}=0.011$ ). Higher LVEF was a factor of better prognosis.

Conclusions: Patients with HFrEF and AFib did not derive advantages from a six-month training program. After a 54-month follow-up there were more all-cause deaths in all AFib patients. Independent risk factors of mortality in multivariate logistic regression analysis were female gender and AFib. Higher LVEF was a factor of better prognosis.

\section{Background}

Due to increasing numbers of patients with heart failure and reduced left ventricular ejection fraction (HFrEF) treated with cardiac resynchronization therapy (CRT), atrial fibrillation (AFib) occurrence may be a potential problem regarding treatment efficacy. AFib prevalence grows proportionally with advanced age, NYHA class and comorbidities in up to $50 \%$ of patients with HFrEF. The CRT defibrillator (CRT-D) is a complementary treatment option in patients with HFrEF. Based on the existing data, its role has been strongly emphasized in the prevention of all-cause mortality and hospitalizations in both the former and current European and American guidelines ${ }^{[1-6]}$. Results of clinical trials showed that HFrEF patients with a wide QRS complex and signs of dyssynchrony had moderate improvement in NYHA functional class, quality of life, exercise capacity (peak oxygen uptake), and left ventricular ejection fraction (LVEF) after effective biventricular (BIV) pacing ${ }^{[7-8]}$. However, in reality, one-third of them do not respond to the CRT-D. The additional option in patients with reduced LVEF is direct prevention of sudden cardiac death by an implantable cardioverter-defibrillator (ICD). The CRT-D may also be recommended initially in HFrEF patients with AFib and coexisting atrioventricular block to avoid the consequences of right ventricular pacing and after atrioventricular junction ablation (class $\mathrm{IIa} / \mathrm{B})^{[6,9]}$.

At present exercise training (ExT) remains an additional and important option in the treatment of patients with HFrEF, including those with cardiovascular implantable electronic devices (CIED). Numerous studies of regular ExT in patients with HFrEF, including those with a CRTD or ICD, confirm improvement in exercise parameters and safety of regular, controlled exercise ${ }^{[6,10-14]}$. Data related to ExT programs' efficacy and further survival are not definitively conclusive for CRT-D patients with AFib. No deleterious effect of AFib in these patients has been established.

The aim of the study was to evaluate the impact of ExT on overall performance and prognosis in patients with HFrEF, NYHA class III, AFib and CIED: CRT-D and ICD.

\section{Methods}

The study was conducted during the period 2008-2014. Inclusion criteria were: HFrEF (LVEF $\leq 35 \%$ ) of ischemic and non-ischemic etiology, CRT-D or ICD a minimum of six weeks after implantation, hospitalization for worsening HFrEF, and no training programs in the past. Patients were randomly assigned to a training program which started in hospital, and which was continued for six months. The average hospital stay was $9 \pm 3$ days. All patients received optimal drug therapy including oral anticoagulants. Exclusion criteria were: acute coronary syndromes; TIA or stroke within the last six months; cardiac surgery or coronary intervention within the last 3 months; uncontrolled hypertensive and valve diseases; acute or uncontrolled non-cardiac diseases; severe musculoskeletal conditions which preclude physical exercise training (orthopedic, neurological); history of venous thrombosis or pulmonary embolism. At discharge and after six months ergospirometry tests (CPX) and standard echo examinations were performed.

All the patients underwent interval cycloergometer endurance workouts and conditioning trainings with elements of resistance exercises in aerobic conditions three times a week, for one hour in a structured, supervised, centre-based program in an in-hospital facility. The ExT was supervised by physiotherapists. A cardiologist was called if necessary. Each patient's heart rate and blood pressure were monitored. Training programs were adjusted according to exercise test results. They were modified due to any patient's discomfort and were performed at the level of $40 \%$ or $3-4 / 10$ on the Borg scale. The intensity of endurance training was based on the maximum heart rate achieved during the exercise test. The patient's pulse during training was calculated according to the Karvonen formula. Cycloergometer sessions lasted 30 minutes. 
Patients were cycling with speeds of 55 to $64 \mathrm{rpm}$. Resistance training sessions lasted from 5 to $10 \mathrm{~min}$. Exercises involved a single muscle group of one limb, alternately, with a load of up to $50 \%$ of muscle strength. During the working phase lasting 1 minute every patient had 10-12 repetitions in one cycle. Patients could not feel fatigue more than 11-12 according to the Borg scale. The training intensity was not dependent on the current beta-blocker and digoxin treatment. The analysis of CPX results included: exercise tolerance (metabolic equivalents, METs); peak oxygen consumption (peak $\mathrm{VO}_{2}$ ); peak carbon dioxide excretion (peak $\mathrm{VCO}_{2}$ ); ventilation anaerobic threshold (VAT) and minute ventilation (VE).

Standard echocardiographic examinations (2D plus Doppler) were performed at baseline and after six months. LVEF was measured by the biplane Simpson method. Beck Depression Inventory (BDI) scoring was employed at baseline and after six months. The follow-up lasted 551-2272, mean 1621 days (54 months), with regular clinical visits in ambulatory conditions every 3-6 months.

The paper presents additional data and analysis of patients with AFib in the study.

The study was supported by a State Committee for Scientific Research grant (NN 404171934). The design and protocol of the study were accepted by the Institutional Review Board (registration number IK-NP-0021-5/996/07, IK-NP-0021-73/1100/08).

\section{Statistical analysis}

Due to the normal distribution of continuous variables verified by the Kolmogorov-Smirnov test, the results were expressed as mean $\pm S D$ and compared by paired (within groups comparison) or non-paired (between groups comparison) Student's t test, and multivariate analysis of variance (MANOVA). Interactions between time and main effects (training group and repeated measurement) were included in the model. Categorical variables were presented as counts and percentages (\%). Differences between groups were verified by the chi-square test or Fisher's exact when the expected cell count was at least 5. Multivariate analysis was performed using regression. Adjusted odds ratios with 95\% intervals and $\mathrm{p}$-values from corresponding Wald statistics have been provided. The probabilities of overall mortality (mortality rate) were estimated by the Kaplan-Meier method and were compared using the log-rank test.

In the two-sided test, a P-value of $<0.05$ was regarded as statistically significant. Statistical analyses were performed using SAS version 9.2 software.

\section{Results}

124 patients were enrolled in the study: 49 with permanent AFib. (31 with CRT-D, 63.3\%) and 75 in sinus rhythm (SR, 46 with CRT-D, $61.3 \%$, ns). Mean age of the AFib group was higher: $65.3 \pm 7.8$ vs. $60.7 \pm 8.2$ years $(p=0.0022$ ). More of the AFib patients had diabetes. Mean baseline HR values from standard baseline ECG are presented in Tables 2 and 3 for analyzed groups. Ischemic etiology of HFrEF was most prevalent in AFib patients $(61.2 \%)$ as well in patients with sinus rhythm $(66.7 \%), p=0.54$. There were no differences in the proportions of subjects in the training program: 22 (44.9\%) with AFib and 38 $(50.7 \%, \mathrm{~ns})$ with SR, started in hospital settings and continued for six months. AFib patients were treated more often with loop diuretics, insulin, oral anticoagulants and less frequently with aspirin (Table 1). There were no differences in other clinical characteristics or ICD interventions. The average BIV capture was $93 \%$ in the AFib and SR group (ns).

Table 1. Demographic and clinical characteristics of the study groups.

\begin{tabular}{|c|c|c|c|}
\hline & $\begin{array}{l}A F(+) \\
n=49(65.3 \%)\end{array}$ & $\begin{array}{l}\text { AF (-) } \\
n=75\end{array}$ & $P$ \\
\hline Age, years & $65.3 \pm 7.8$ & $60.7 \pm 8.2$ & 0.0022 \\
\hline Male, n $[\%]$ & $46(93.9 \%)$ & $66(88.0 \%)$ & 0.36 \\
\hline Body mass, kg & $86.7 \pm 17.0$ & $81.7 \pm 15.9$ & 0.10 \\
\hline $\mathrm{BMI}, \mathrm{kg} / \mathrm{m} 2$ & $29.1 \pm 5.0$ & $27.7 \pm 4.5$ & 0.10 \\
\hline $\operatorname{LVEF}(\%):$ & $24.1 \pm 4.9$ & $24.8 \pm 8.0$ & 0.62 \\
\hline LVDD & $6.67 \pm 0.85$ & $6.73 \pm 0.92$ & 0.70 \\
\hline $\mathrm{HR}$ & $72.9 \pm 18.3$ & $67.4 \pm 13.9$ & 0.10 \\
\hline CRT, n [\%] & 31 (63.3) & $46(61.3)$ & 0.82 \\
\hline ICD, $\mathrm{n}[\%]$ & $18(36.7)$ & $29(38.7)$ & 0.82 \\
\hline Training program & $22(44.9)$ & $38(50.7)$ & 0.52 \\
\hline CRT training program & $16(51.6)$ & $28(60.9)$ & 0.42 \\
\hline ICD training program & 6 (33.3) & 10 (34.5) & 0.93 \\
\hline Coronary disease, $\mathrm{n}(\%)$ & $30(61.2)$ & $50(66.7)$ & 0.54 \\
\hline Myocardial infarction, n [\%] & 25 (51.0) & $41(59.4)$ & 0.36 \\
\hline Arterial hypertension, $\mathrm{n}[\%]$ & $22(44.9)$ & $35(50.7)$ & 0.53 \\
\hline Diabetes mellitus, n (\%) & $20(40.8)$ & 14 (20.3) & 0.0153 \\
\hline Beck depression questionnaire & $11.73 \pm 7.3$ & $11.45 \pm 7.3$ & 0.83 \\
\hline Beta-blockers, n (\%) & 49 (100) & 75 (100) & NA \\
\hline ACE-Inhibitors, n (\%) & 43 (87.8) & $56(74.7)$ & 0.07 \\
\hline ARB, n (\%) & $7(14.3)$ & $16(21.3)$ & 0.32 \\
\hline Loop diuretics, n (\%) & $44(89.8)$ & 53 (70.7) & 0.0116 \\
\hline Thiazide diuretics, n (\%) & $7(14.4)$ & $8(10.7)$ & 0.54 \\
\hline Spironolactone, $\mathrm{n}(\%)$ & 32 (65.3) & 55 (73.9) & 0.33 \\
\hline Eplerenone, n [\%] & $8(16.3)$ & 10 (13.3) & 0.64 \\
\hline Statins, n $[\%]$ & $34(69.4)$ & 53 (70.7) & 0.87 \\
\hline Fibrates, n (\%) & $2(4.1)$ & $8(10.7)$ & 0.31 \\
\hline Digoxin, $\mathrm{n}(\%)$ & 19 (38.8) & $12(16.0)$ & 0.0042 \\
\hline Oral anticoagulants, $\mathrm{n}(\%)$ & $37(75.5)$ & $11(14.7)$ & $<0.0001$ \\
\hline Oral antidiabetic agents, $\mathrm{n}(\%)$ & $9(18.4)$ & $13(17.3)$ & 0.88 \\
\hline Insulin, n (\%) & $8(16.3)$ & $2(2.7)$ & 0.0137 \\
\hline ASA, n (\%) & 19 (38.8) & $56(74.7)$ & $<0.0001$ \\
\hline Ticlopidin, n (\%) & 0 & $3(4.0)$ & 0.27 \\
\hline Clopidogrel, n (\%) & $8(16.3 \%)$ & 10 (13.3) & 0.64 \\
\hline
\end{tabular}

BMI: body mass index; LVEF: left ventricular ejection fraction; CRT: cardiac resynchronisation therapy; ICD: implantable cardioverter defibrillator; ACE-I: angiotensin converting enzyme-inhibitors; ARB: angiotensin receptor blockers; ASA: aspirin. 
Table 2. Echocardiographic and CPX parameters at baseline and after six months in patients with atrial fibrillation (AFib).

\begin{tabular}{|c|c|c|c|c|c|c|c|c|c|c|c|}
\hline & \multicolumn{3}{|c|}{ Training $(+), n=22$} & \multicolumn{3}{|c|}{ Training $(-), n=27$} & \multicolumn{2}{|c|}{$\begin{array}{c}\text { Training }(+) \text { vs. Training } \\
(-)\end{array}$} & \multicolumn{3}{|c|}{ MANOVA, $p$} \\
\hline & Baseline & 6 months & $P$ & Baseline & 6 months & $P$ & Baseline & 6 months & Time & Rehab & $\begin{array}{l}\text { Time * } \\
\text { rehab }\end{array}$ \\
\hline LVEDD (cm) & $6.92 \pm 0.92$ & $6.60 \pm 1.00$ & 0.0797 & $6.43 \pm 0.74$ & $6.34 \pm 0.66$ & 0.58 & 0.0569 & 0.31 & 0.58 & 0.09 & 0.33 \\
\hline LVESD (cm) & $5.76 \pm 1.03$ & $4.94 \pm 1.12$ & 0.0394 & $5.22 \pm 1.05$ & $4.43 \pm 0.88$ & 0.0379 & 0.1509 & 0.14 & 0.0243 & 0.0428 & 0.96 \\
\hline RVEDD (cm) & $2.71 \pm 0.54$ & $3.09 \pm 0.61$ & 0.0120 & $3.00 \pm 0.84$ & $3.26 \pm 0.54$ & 0.05 & 0.2205 & 0.38 & 0.05 & 0.23 & 0.50 \\
\hline LA $(\mathrm{cm})$ & $4.82 \pm 0.61$ & $4.97 \pm 0.84$ & 0.3799 & $4.93 \pm 0.76$ & $4.63 \pm 0.58$ & 0.08 & 0.5978 & 0.12 & 0.06 & 0.52 & 0.06 \\
\hline Aorta $(\mathrm{cm})$ & $3.58 \pm 0.33$ & $3.56 \pm 0.38$ & 0.8126 & $3.60 \pm 0.45$ & $3.61 \pm 0.39$ & 0.84 & 0.8765 & 0.66 & 0.81 & 0.73 & 0.77 \\
\hline LVEF (\%) & $23.0 \pm 3.8$ & $28.6 \pm 8.0$ & 0.0005 & $25.0 \pm 5.8$ & $31.1 \pm 10.1$ & 0.0045 & 0.1916 & 0.38 & 0.0007 & 0.25 & 0.83 \\
\hline $\mathrm{VO}_{2}(\mathrm{l} / \mathrm{min})$ & $12.2 \pm 2.5$ & $12.3 \pm 4.2$ & 0.9377 & $11.2 \pm 4.7$ & $11.7 \pm 4.1$ & 0.67 & 0.4418 & 0.68 & 0.64 & 0.47 & 0.79 \\
\hline $\mathrm{VO}_{2} \%$ & $52.6 \pm 14.4$ & $53.5 \pm 17.9$ & 0.8451 & $47.0 \pm 20.8$ & $51.0 \pm 19.3$ & 0.39 & 0.3621 & 0.69 & 0.35 & 0.44 & 0.63 \\
\hline $\begin{array}{l}\mathrm{ATVO}_{2}(\mathrm{ml} / \mathrm{kg} / \\
\mathrm{min})\end{array}$ & $12.4 \pm 1.4$ & $11.4 \pm 5.0$ & 0.7175 & $7.6 \pm 2.8$ & $11.4 \pm 5.6$ & 0.12 & 0.0119 & 0.99 & 0.16 & 0.30 & 0.18 \\
\hline $\mathrm{ATVO}_{2} \%$ & $61.8 \pm 17.1$ & $57.8 \pm 34.0$ & 0.7262 & $66.5 \pm 36.2$ & $48.7 \pm 20.8$ & 0.42 & 0.8026 & 0.65 & 0.28 & 0.89 & 0.52 \\
\hline $\mathrm{HR} / \mathrm{min}$ & $69.4 \pm 17.6$ & $69.5 \pm 18.3$ & 0.9878 & $76.9 \pm 21.2$ & $72.2 \pm 15.1$ & 0.26 & 0.2710 & 0.64 & 0.23 & 0.35 & 0.41 \\
\hline $\begin{array}{l}\text { METs (ml/kg/ } \\
\min )\end{array}$ & $3.5 \pm 1.2$ & $4.5 \pm 2.3$ & 0.0588 & $3.2 \pm 1.3$ & $3.5 \pm 1.7$ & 0.38 & 0.4718 & 0.09 & 0.51 & 0.12 & 0.19 \\
\hline BDI & $11.8 \pm 8.5$ & $7.9 \pm 4.6$ & 0.0232 & $12.2 \pm 6.5$ & $10.8 \pm 9.0$ & 0.52 & 0.8462 & 0.16 & 0.45 & 0.18 & 0.31 \\
\hline
\end{tabular}

CPX: cardiopulmonary exercise testing; Rehab: rehabilitation program; LVEDD: left ventricular end-diastolic dimension; LVESD: left ventricular end-systolic dimension; RVEDD: right ventricular end-diastolic dimension; LA: left atrial end-systolic diameter; Aorta: ascending aorta diameter; LVEF: left ventricular ejection fraction; VO peak: peak oxygen uptake; ATVO2: anaerobic threshold; HR: heart rate; METs: metabolic equivalents; BDI: Beck depression inventory.

Table 3. Echocardiographic and CPX parameters at baseline and after 6 months in the group of patients in sinus rhythm.

\begin{tabular}{|c|c|c|c|c|c|c|c|c|c|c|c|}
\hline & \multicolumn{3}{|c|}{ Training $(+), n=38$} & \multicolumn{3}{|c|}{ Training (-), $n=37$} & \multicolumn{2}{|c|}{$\begin{array}{c}\text { Training (+) vs. Training } \\
(-)\end{array}$} & \multicolumn{3}{|c|}{ MANOVA, $p$} \\
\hline & Baseline & 6 months & $P$ & Baseline & 6 months & $P$ & Baseline & 6 months & Time & Rehab & $\begin{array}{l}\text { Time* } \\
\text { rehab }\end{array}$ \\
\hline LVEDD (cm) & $6.51 \pm 0.80$ & $6.19 \pm 0.99$ & 0.0457 & $6.79 \pm 1.035$ & $6.44 \pm 0.99$ & 0.0377 & 0.23 & 0.32 & 0.0289 & 0.22 & 0.86 \\
\hline LVESD (cm) & $4.99 \pm 1.04$ & $4.07 \pm 0.99$ & 0.0010 & $5.36 \pm 1.20$ & $4.96 \pm 1.10$ & 0.0434 & 0.25 & 0.0048 & 0.05 & 0.0256 & 0.09 \\
\hline RVEDD (cm) & $2.78 \pm 0.64$ & $3.15 \pm 0.57$ & 0.0573 & $2.59 \pm 0.77$ & $2.98 \pm 0.83$ & 0.0059 & 0.32 & 0.38 & 0.0113 & 0.26 & 0.92 \\
\hline $\mathrm{LA}(\mathrm{cm})$ & $4.70 \pm 0.61$ & $4.53 \pm 0.57$ & 0.15 & $4.48 \pm 0.82$ & $4.57 \pm 0.98$ & 0.55 & 0.27 & 0.84 & 0.51 & 0.64 & 0.16 \\
\hline Aorta $(\mathrm{cm})$ & $3.50 \pm 0.58$ & $3.49 \pm 0.48$ & 0.87 & $3.38 \pm 0.39$ & $3.40 \pm 0.35$ & 0.64 & 0.32 & 0.44 & 0.70 & 0.33 & 0.68 \\
\hline LVEF (\%) & $26.2 \pm 7.2$ & $31.1 \pm 8.7$ & 0.0002 & $24.5 \pm 8.6$ & $29.5 \pm 10.4$ & 0.0025 & 0.39 & 0.50 & 0.0006 & 0.40 & 0.96 \\
\hline $\mathrm{VO}_{2}(\mathrm{l} / \mathrm{min})$ & $14.2 \pm 4.4$ & $15.5 \pm 4.9$ & 0.15 & $13.5 \pm 4.1$ & $14.3 \pm 3.8$ & 0.40 & 0.57 & 0.33 & 0.40 & 0.35 & 0.68 \\
\hline $\mathrm{VO}_{2} \%$ & $54.1 \pm 16.0$ & $60.2 \pm 19.8$ & 0.09 & $51.5 \pm 16.6$ & $57.5 \pm 20.8$ & 0.12 & 0.55 & 0.62 & 0.11 & 0.53 & 0.97 \\
\hline $\begin{array}{l}\operatorname{ATVO}_{2}(\mathrm{ml} / \mathrm{kg} / \\
\mathrm{min})\end{array}$ & $11.9 \pm 3.3$ & $13.0 \pm 4.3$ & 0.28 & $13.5 \pm 2.8$ & $11.7 \pm 4.5$ & 0.24 & 0.18 & 0.44 & 0.18 & 0.89 & 0.10 \\
\hline $\mathrm{ATVO}_{2} \%$ & $60.1 \pm 23.3$ & $55.3 \pm 21.5$ & 0.48 & $67.8 \pm 20.7$ & $47.7 \pm 18.5$ & 0.09 & 0.38 & 0.35 & 0.0391 & 0.98 & 0.21 \\
\hline $\mathrm{HR} / \mathrm{min}$. & $67.5 \pm 11.9$ & $73.2 \pm 13.3$ & 0.0337 & $67.5 \pm 13.1$ & $68.7 \pm 15.1$ & 0.63 & 0.99 & 0.25 & 0.64 & 0.48 & 0.21 \\
\hline $\begin{array}{l}\text { METs (ml/kg/ } \\
\text { min) }\end{array}$ & $4.3 \pm 2.0$ & $5.4 \pm 2.2$ & 0.0062 & $3.3 \pm 1.7$ & $4.2 \pm 1.8$ & 0.0105 & 0.0334 & 0.0368 & 0.0125 & 0.0171 & 0.82 \\
\hline BDI & $11.7 \pm 6.2$ & $9.2 \pm 5.5$ & 0.0061 & $11.5 \pm 7.3$ & $11.2 \pm 6.1$ & 0.82 & 0.88 & 0.16 & 0.71 & 0.88 & 0.12 \\
\hline
\end{tabular}

CPX: cardiopulmonary exercise testing; Rehab: rehabilitation program; LVEDD: left ventricular end-diastolic dimension; LVESD: left ventricular end-systolic dimension; RVEDD: right ventricular end-diastolic dimension; LA: left atrial end-systolic diameter; Aorta: ascending aorta diameter; LVEF: left ventricular ejection fraction; VO peak: peak oxygen uptake; $\mathrm{ATVO}_{2}$ : anaerobic threshold; HR: heart rate; METs: metabolic equivalents; BDI: Beck depression inventory.

After six months most of the CPX parameters in the AFib group subjects who underwent exercise training, and those who did not, had not significantly changed. In SR patients only exercise tolerance (METs) improved, proportionally to baseline values (Tables 2, 3).
Relative change in $\mathrm{VO}_{2}$ evaluation after 6 months in $\mathrm{AF}$ patients was $0.8 \%$ in the exercise training group and $4.5 \%$ in the control group. Relative change in $\mathrm{VO}_{2}$ evaluation after 6 months in sinus rhythm patients was $9.2 \%$ in the exercise training group and $5.9 \%$ in the control group. 
After six months mean LVEF in the AFib patients significantly increased while left ventricular end-systolic dimension (LVESD) was reduced. In SR patients LVEF both in training and non-training patients also increased while LVESD and LVEDD were reduced in training and non-training patients. Right ventricle dimensions increased significantly in the AFib training group (Table 2). There was an improvement in the Beck Depression Inventory scoring only in those patients who had participated in a training program both in AFib and SR groups (Table 2).

In the additional analysis of patients with AFib and CRT-D who exercised and who did not, CPX parameters did not change significantly in either group.

\section{Long-term survival}

In a 54-month (mean 1621 days) follow-up 36 patients died. Due to insufficient data about mode of deaths (26 outside the hospital), we did not analyze this issue and we concentrated on all-cause deaths. The overall survival rate was 0.75 and was significantly longer among the 75 patients who did not have AFib (0.80) than among the 49 patients with AFib (0.63, log-rank $\mathrm{p}=0.0098$ ). Of the 77 patients who had a CRT-D implanted, the survival rate was 0.60 in the AFib group, and 0.83 in the no-AFib group (log-rank $p=0.0259$ ). Of the 47 patients who had an ICD implanted, survival rates were the same regardless of the presence of AFib (0.66, 0.67, log-rank $p=0.58)$. Death-independent risk factors in logistic regression analysis were female gender (adjusted OR 13.2, 95\% CI 1.95-86.1, $\mathrm{p}=0.008$ ) and atrial fibrillation (adjusted OR $5.13,95 \%$ CI 1.4518.1, $\mathrm{p}=0.011$ ). Higher LVEF was a factor of better prognosis.

All-cause deaths in patients with CRT-D occurred in 12 (38.7\%) of the AFib group and in 7 (15.2\%) of the SR group, $\mathrm{p}=0.019$. However, no difference in ICD patients was found: 8 $(44.4 \%)$ in the AFib group vs. 9 (31.0\%) in the SR group, $p=0.35$. Figure 1 shows Kaplan-Meier estimates of the survival rate according to the AFib group and rehabilitation strategy. The survival rate was significantly higher in the SR group who underwent exercise training than in AFib patients who were training (log rank test: 0.0033).

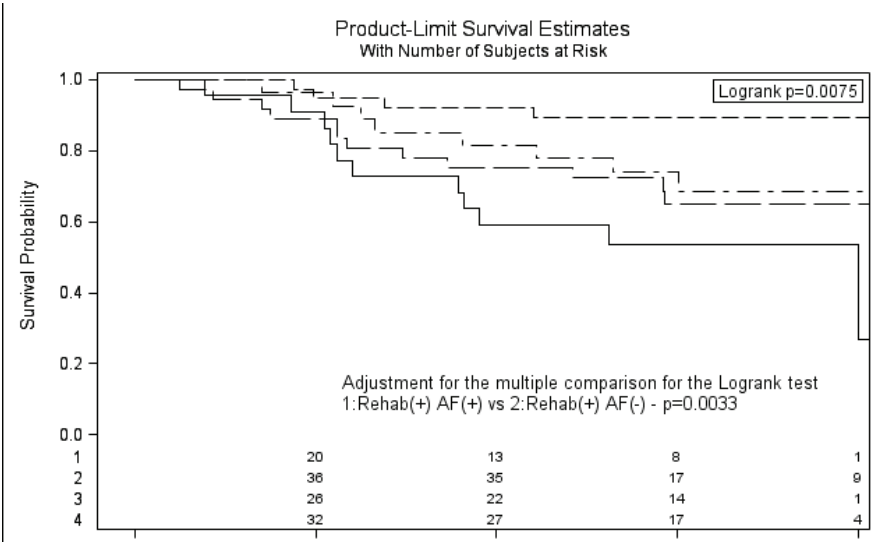

Figure 2. Kaplan-Meier curves of survival probability for patients with AFib and without AFib, with or without exercise training.

\section{Discussion}

The results of the present study show that training programs in advanced heart failure patients, NYHA III class, with
AFib and CRT-D may not be beneficial. AFib significantly impairs cardiac function, which results in 20-30\% reduction of stroke volume, which is important in HFrEF patients. It is possible that the worse results of ExT and prognosis in the CRT-D group can be explained due to the suboptimal percentage of BIV stimulation (average 93\%) in spite of optimal medical therapy: $100 \%$ of patients were on beta-blockers and $33.8 \%$ on digoxin. A lot of data support the evidence for the benefits of CRT-D in HFrEF patients. In the BLOCK HF trial BIV pacing was superior to conventional right ventricular pacing in patients with atrioventricular block, left ventricular systolic dysfunction and heart failure, NYHA class I-III ${ }^{[9]}$. The clinical efficacy of CRT-D depends on complete and consistent BIV capture. In the present study no patient had atrioventricular node ablation (AVNA). The average BIV capture was 93\% in both groups. In the CERTIFY registry of 443 patients with CRT and AFib, the AVNA procedure was associated with better outcomes than treatment with rate-slowing drugs. In multivariable analysis total and cardiac mortality were similar for patients with AFib and AVNA and patients in SR in 37 months of follow-up ${ }^{[15]}$. The results of the MILOS registry showed that patients with HFrEF and AFib treated with CRT-D had similar mortality rates in comparison with patients in SR. AVNA in addition to CRT-D significantly improved overall survival compared with CRT-D alone, primarily by reducing heart failure deaths in patients with HFrEF and AFib ${ }^{[16]}$. The conclusion from another study was that CRT-D in permanent AFib patients showed large and sustained long-term improvement of left ventricular function and functional capacity, similar to patients in SR, only if AVNA ablation was performed ${ }^{[17]}$. A meta-analysis of five prospective cohort studies including a total of 1164 patients compared the impact of CRT-D in patients with AFib and SR. Both AFib and SR patients benefited significantly from CRT-D, but to a lesser degree in regard to functional outcomes. Mortality was not significantly different at 1 year (relative risk ratio: $1.57,95 \%, \mathrm{CI}: 0.87$ to 2.81 ). Patients in SR showed greater relative improvement in the 6-minute walk test (6-MWT) than AFib patients ${ }^{[18]}$. These results are consistent with our findings, but in the present study physical condition was evaluated more precisely by cardiopulmonary exercise testing. The MUltisite STimulation in Cardiomyopathies (MUSTIC) trial demonstrated similar improvement in the 6-MWT in class III HFrEF patients following CRT whether they were in SR or had AFib. All the patients with AFib had a slow ventricular rate due to either spontaneous or induced AV block. This likely distinguished a subset of patients with AFib who benefited from CRT due to a consistent high degree of BIV capture ${ }^{[19]}$.

In the CARE-HF trial comparing CRT to pharmacological therapy alone, though mortality was higher among patients who developed new AFib, during the follow-up patients with new AFib benefitted from the CRT-D for all major study end points ${ }^{[7]}$.

In contrast, a prospective observational study of 295 patients with HFrEF and AFib ( $\mathrm{n}=86$, permanent and paroxysmal) who were treated with CRT without AVNA revealed no differences in a composite end point of cardiovascular deaths and hospitalizations for HFrEF after a 7-year follow-up. 
The improvements in NYHA class, 6-MWT and quality of life scores were similar in both groups ${ }^{[20]}$. In the CArdiac REsynchronisation in Heart Failure (CARE-HF) trial, after a 29.4-month follow-up, mortality was higher in patients who developed AFib. However, AFib was not a predictor in the multivariable model ${ }^{[21]}$.

The results of a meta-analysis of 23 observational studies ( $\mathrm{n}=7495$ CRT patients, 33-month follow-up) showed that AFib diagnosis was associated with an increased risk of non-response to CRT-D (RR 1.32; 95\% CI, p=0.001) and all-cause mortality ( $10.8 \%$ vs. $7.1 \%$ per year; $R R, 1.50 ; 95 \% \mathrm{CI}, \mathrm{p}=0.015)$. The presence of AFib was additionally associated with a smaller improvement in quality of life and 6-MWT ${ }^{[22]}$. These data were also confirmed in another meta-analysis which included 19 studies involving 5324 patients $^{[23]}$. According to the heart failure guidelines, CRT-D in patients with AFib and HFrEF may be considered with the option of AVNA, which ensures obligate ventricular pacing $(\mathrm{IIaB})^{[6]}$. What is important is that there are no definitive data showing that CRT-D may decrease the incidence of new-onset AFib. In an observational study AFib occurred in $27 \%$ of patients during a 5-year follow-up ${ }^{[24]}$.

On the other hand, in a prospective trial (RAFT) of patients with permanent AFib and HFrEF randomized to CRT-D $(\mathrm{n}=114)$ or ICD $(\mathrm{n}=115)$ no difference in the primary outcome (death) or heart failure hospitalization after 40 months was observed. The change in 6-MWT duration between baseline and 12 months was also not different between treatment arms. There was no clear benefit of CRT in AFib patients ${ }^{[25]}$.

According to our results, AFib, as an independent mortality risk factor in heart failure patients with CRT, was identified in other former studies ${ }^{[26,27]}$. In the present study, gender was another mortality risk factor, stronger than AFib, which is a novel finding contrary to the results of previous studies. But, in these studies, only $22 \%$ and $26 \%$ were female patients ${ }^{[28,29,30]}$. In our study the risk of death in women was 13 times higher than in men and mortality risk due to AFib presence was 5.1 times higher than in patients without AFib. It is difficult to explain this phenomenon because there were no significant differences in comparison to men in baseline characteristics. The lower survival rate of AFib and CRTD patients may be due to a worse initial clinical condition which implicated CRT-D implantation and promoted AFib occurrence. It is well proven that AFib patients are more sick than patients with SR - they have more comorbidities and they deserve more intensive heart failure treatment - as in the present study. This may have additionally influenced the outcomes.

Table 4. Logistic regression analysis - identification of independent all cause mortality risk

Odds ratio $\pm 95 \% \mathrm{Cl} \quad \mathrm{P}$

Without variables selection $(\mathbf{A U C}=\mathbf{0 . 8 5}$ )

\begin{tabular}{|l|l|l|}
\hline Age & $0.98[0.91-1.04]$ & 0.46 \\
\hline Sex (women vs. men) & $\mathbf{1 3 . 0}[\mathbf{1 . 9}-\mathbf{8 6 . 1}]$ & $\mathbf{0 . 0 0 8}$ \\
\hline Atrial fibrillation & $\mathbf{5 . 1}[\mathbf{1 . 4}-\mathbf{1 8 . 1}]$ & $\mathbf{0 . 0 1 1}$ \\
\hline Training yes vs. no & $1.00[0.34-2.94]$ & 0.99 \\
\hline ICD vs. CRT-D & $1.78[0.62-5.13]$ & 0.28 \\
\hline
\end{tabular}

\begin{tabular}{|l|l|l|}
\hline Ejection fraction & $\mathbf{0 . 7 9}[\mathbf{0 . 7 1}-\mathbf{0 . 8 9}]$ & $<\mathbf{0 . 0 0 0 1}$ \\
\hline Diabetes & $\mathbf{0 . 9 9}[\mathbf{0 . 3 0 - 3 . 2 6 ]}$ & $\mathbf{0 . 9 9}$ \\
\hline \multicolumn{3}{|c|}{ With procedure: 'stepwise' as a method of variables selection } \\
[AUC=0.8] \\
\hline Sex (women vs. men] & $13.2[1.9-86.1]$ & 0.008 \\
\hline Atrial fibrillation & $5.1[1.45-18.1]$ & 0.011 \\
\hline Ejection fraction & $0.79[0.70-0.88]$ & $<0.0001$ \\
\hline
\end{tabular}

$\mathrm{Cl}$ - confidence interval, AUC - area under curve, ICD - implantable cardioverter-defibrillator, CRT-D - cardiac resynchronization therapy with cardioverter function.

A novel aspect of this study is the evaluation of results in AFib patients with CRT-D by cardiopulmonary testing application. CPX is a more precise method of clinical and physiological evaluation than a 6-minute walk test for HFrEF patients, NYHA class III. The question of training programs' implications in AFib patients with HFrEF and CRT-D, NYHA class III, remains open. Even in patients with sinus rhythm, consistent pacing and capture is difficult to achieve. Fusion and pseudo-fusion beats, accelerated ventricular rates during activity, frequent ventricular arrhythmia in patients with low ejection fraction - all of these may be responsible for ineffective pacing ${ }^{[31,32,33]}$. The optimistic finding is fewer depression symptoms in exercising HFrEF patients, including those with AFib.

The concept of possible successful AFib ablation (pulmonary vein isolation) proposed by some authors is controversial due to the growing number of arrhythmia substrates in heart failure patients which limits procedure efficacy in the longer term perspective, although the results of the CASTLE-AF study are optimistic ${ }^{[34,35]}$. Potentially beneficial but still limited impact of tele-monitoring to enhance the care in many clinical aspects in cardiac devices recipients should be taken into account ${ }^{[36]}$.

Another problem is tailored, regular, daily exercise activity, which may result in lowering of BIV capture in patients without AVNA, which paradoxically may lead to deterioration of cardiac performance, which in the longer term may influence prognosis.

The limitations of the present study are the one-centre design and a relatively small number of patients. On the other hand, it is challenging to find stable NYHA III heart failure patients willing, after fulfilling inclusion criteria, to take part in a 6-month exercise training program.

\section{Conclusions}

Patients with HFrEF and AFib did not derive advantages from a six-month exercise training program. After a 54-month follow-up there were more all-causes deaths in all AFib patients and those with AFib and CRT-D. Independent risk factors of mortality in multivariate logistic analysis were female gender and atrial fibrillation. Higher LVEF was a factor of better prognosis. 


\section{References}

1. Vardas PE, Auricchio A, Blanc JJ, et al. Guidelines for cardiac pacing and cardiac resynchronization therapy. Eur Heart J 2007; 28: 2256-2295.

2. Dickstein K, Cohen-Solal A, Filippatos G, et al. ESC Guidelines for the diagnosis and treatment of acute and chronicheartfailure2008. EurJHeartFail 2008; 10:933-989.

3. Dickstein K, Vardas PE, Auricchio A, et al. 2010 Focused Update of ESC Guidelines on device therapy in heart failure: an update of the 2008 ESC Guidelines for the diagnosis and treatment of acute and chronic heart failure and the 2007 ESC guidelines for cardiac and resynchronization therapy. Eur Heart J 2010; 31: 2677-2687.

4. Yancy CW, Jessup M, Bozkurt B, et al. 2013 ACCF/AHA Guideline for the Management of Heart Failure. Circulation 2013; 128: 240-327.

5. Priori SG, Blomstrom-Lundqvist CB, Mazzanti A et al. 2015 ESC Guidelines for the management of patients with ventricular arrhythmias and the prevention of sudden cardiac death. Eur Heart J 2015; 36: 2793-2867.

6. Ponikowski P, Voors AA, Anker SD, at al. 2016 ESC Guidelines for the diagnosis and treatment of acute and chronic heart failure. Eur Heart J 2016; 37: 2129-2200.

7. Cleland JG, Daubert JC, Erdmann E, et al. The effect of cardiac resynchronization on morbidity and mortality in heart failure. Cardiac Resynchronization-Heart Failure (CARE-HF) Study Investigators. N Engl J Med 2005; 352: 1539-1549.

8. Moss AJ, Hall WJ, Cannon DS, et al. For the MADIT-CRT Trial Investigators. Cardiac-resynchronization therapy for the prevention of heart-failure events. N Engl J Med 2009; 361: 1329-1338.

9. Curtis AB, Worley SJ, Adamson PB, et al. Biventricular versus Right Ventricular Pacing in Heart Failure Patients with Atrioventricular Block (BLOCK HF) Trial Investigators. N Engl J Med 2013; 369: 1585-1593.

10. O'Connor CM, Whellan DJ, Lee KL, et al. for the HF-ACTION Investigators. Efficacy and safety of exercise training in patients with chronic heart failure: HF-ACTION Randomized Controlled Trial. JAMA 2009; 301: 1439-1450.

11. Flynn KE, Piña IL, Whellan DJ, et al. Effects of exercise training on health status in patients with chronic heart failure: findings from the HF-ACTION randomized controlled trial. JAMA 2009; 301: 1451-1459.
12. Belardinelli R, Capestro F, Misiani A, et al. Moderate exercise training improves functional capacity, quality of life, and endothelium-dependent vasodilation in chronic heart failure patients with implantable cardioverter defibrillators and cardiac resynchronization therapy. Eur J Cardiovasc Prev Rehabil 2006;13: 818-825.

13. Conraads V, Vanderheyden M, Paelinck B, et al. The effect of endurance training on exercise capacity following cardiac resynchronization therapy in chronic heart failure patients: a pilot trial. Eur J Cardiovasc Prev Rehabil 2007; 14: 99-106.

14. Patwala A, Woods P, Sharp L, et al. Maximizing patient benefit from cardiac resynchronization therapy with the addition of structured exercise training J Am Coll Cardiol 2009; 53: 2332-2339.

15. Gasparini M, Leclercq C, Lunati M, et al. Cardiac resynchronization therapy in patients with atrial fibrillation. The CERTIFY study (Cardiac Resynchronization Therapy in Atrial Fibrillation Patients Multinational Registry). JACC Heart Fail 2013; 1: 500-507.

16. Gasparini M, Auricchio A, Metra M, et al. Long-term survival in patients undergoing cardiac resynchronization therapy: the importance of performing atrio-ventricular junction ablation in patients with permanent atrial fibrillation. Eur Heart J 2008; 29: 1644-1652.

17. Gasparini M, Auricchio A, Regoli F, et al. Fouryear efficacy of cardiac resynchronization therapy on exercise tolerance and disease progression: the importance of performing atrioventricular junction ablation in patients with atrial fibrillation. J Am Coll Cardiol 2006; 48: 734-743.

18. Upadhyay GA, Choudhry NK, Auricchio A, et al. Cardiac resynchronization in patients with atrial fibrillation: a meta-analysis of prospective cohort studies. J Am Coll Cardiol. 2008; 52: 1239-1246.

19. Linde C, Leclercq C, Rex S, et al. Long-term benefits of biventricular pacing in congestive heart failure: results from the MUltisite STimulation in cardiomyopathy (MUSTIC) study. J Am Coll Cardiol 2002; 40: 111-118.

20. Khadjooi K, Foley PW, Chalil S, et al. Long term effects of cardiac resynchronization therapy in patients with atrial fibrillation. Heart 2008; 94: 879-883.

21. Hoppe UC, Casares JM, Eiskjaer H, et al. Effect of cardiac resynchronization on the incidence of atrial fibrillation in patients with severe heart failure. Circulation 2006; 114: 18-25.

22. Wilton SB, Leung AA, et al. Outcomes of cardiac resynchronization therapy in patients with versus 
those without atrial fibrillation: a systematic review and metaanalysis. Heart Rhythm 2011; 8: 1088-1094.

23. Lopes C, Pereira T, Barra S. Cardiac resynchronization therapy in patients with atrial fibrillation: A meta-analysis. Rev Port Cardiol 2014; 33: 717-725.

24. Sade LE, Atar I, Özin B, et al. Determinants of New Onset Atrial Fibrillation in Patients Receiving CRT: Mechanistic Insights From Speckle Tracking Imaging. JACC Cardiovasc Imaging 2016; 9: 99-111.

25. Healey JS, Hohnloser SH, Exner DV, et al. Results from the Resynchronization for Ambulatory Heart Failure Trial (RAFT). Circ Heart Fail 2012; 5: 566-570.

26. van Boven N, Theuns D, Bogaard K, et al. Atrial fibrillation in cardiac resynchronization therapy with a defibrillator: a risk factor for mortality, appropriate and inappropriate shocks. J Cardiovasc Electrophysiol 2013; 24: 1116-1122.

27. Khatib M, Tolosana JM, Trucco E, et al. EAARN score, a predictive score for mortality in patients receiving cardiac resynchronization therapy based on pre-implantation risk factors. Eur J Heart Fail 2014; 16: 802-809.

28. Leyva F, Foley PW, ChalilS, et al. Female gender is associated with a better outcome after cardiac resynchronization therapy. Pacing Clin Electrophysiol 2011;34: 82-88.

29. Zabarovskaja S, Gadler F, Braunschweig F, et al. Women have better long-term prognosis than men after cardiacresynchronization therapy. Europace 2012; 14: 1148-1155.

30. Schuchert A, Muto C, Maounis T, et al. MASCOT Study Group. Gender-related safety and efficacy of cardiac resynchronization therapy. Clin Cardiol 2013; 36: 683-690.

31. Gasparini M, Regoli F, Galimberti P, et al. Cardiac resynchronization therapy in heart failure patients with atrial fibrillation. Europace 2009; 11 Suppl 5: 82-86.

32. Koneru JN, Steinberg JS. Cardiac resynchronization therapy in the setting of permanent atrial fibrillation and heart failure. Curr Opin Cardiol 2008; 23: 9-15.

33. Melenovsky V, Hay I, Fetics BJ, et al. Functional impact of rate irregularity in patients with heart failure and atrial fibrillation receiving cardiac resynchronization therapy. Eur Heart J 2005; 26: 705-711.

34. Sharma AK, Heist K. The utility of cardiac resynchronization therapy in patients with atrial fibrillation. The Journal of Innovations in Cardiac Rhythm Management 2012; 3: 621-626.
35. Marrouche NF, Brachmann J, Andresen D, at al. Catheter ablation for atrial fibrillation with heart failure. N Engl J Med 2018; 378: 417-427.

36. Buchta P, Tajstra M, Kurek A et al. The impact of remote monitoring of implanted cardioverter-defibrillator (ICD) and cardiac resynchronisation therapy device (CRT-D) patients on healthcare costs in the Silesian population: three-year follow-up. Kardiol Pol. 2017;75(6):573-580. 